\title{
How to extract weak values from a mesoscopic electronic system
}

\author{
Iliya Esin • Alessandro Romito · Yuval \\ Gefen
}

Received: date / Accepted: date

\begin{abstract}
Weak value (WV) protocols may lead to extreme expectation values that are larger than the corresponding orthodox expectation values. Recent works have proposed to implement this concept in nano-scale electronic systems. Here we address the issue of how one calibrates the setup in question, maximizes the measurement's sensitivity, and extracts distinctly large WVs. Our concrete setup consists of two Mach-Zehnder interferometers (MZIs): the "system" and the "detector". Such setups have already been implemented in experiment.
\end{abstract}

Keywords weak value $\cdot$ weak measurement $\cdot$ Mach-Zehnder interferometer

\section{Introduction and motivation}

Strong measurement in quantum mechanics leads to the collapse of the measured system's wave function [16]. The challenge of performing a non-invasive measurement is interesting both from the view point of foundations of quantum mechanics, and for concrete applications (e.g. quantum computation). Weakly measuring an observable, while weakly disturbing the system, provides only partial information on the state of the latter. Weak measurements, due to their backaction, can be exploited for quantum feedback schemes [28, 24, 26] and conditional measurements. The latter entail WV protocols 1 and derivatives thereof.

A standard two-step WV protocol consists of a weak measurement step (of the observable $A$ ), followed by a strong one (of $B$ ), $[A, B] \neq 0$. The outcome of the first is conditional on the result of the second (postselection), i.e., one admits the

\section{I.Esin}

Department of Condensed Matter Physics, The Weizmann Institute of Science, Rehovot 76100, Israel

A. Romito

Department of Physics, Lancaster University, Lancaster, LA1 4YB, United Kingdom

Y. Gefen

Department of Condensed Matter Physics, The Weizmann Institute of Science, Rehovot 76100, Israel 


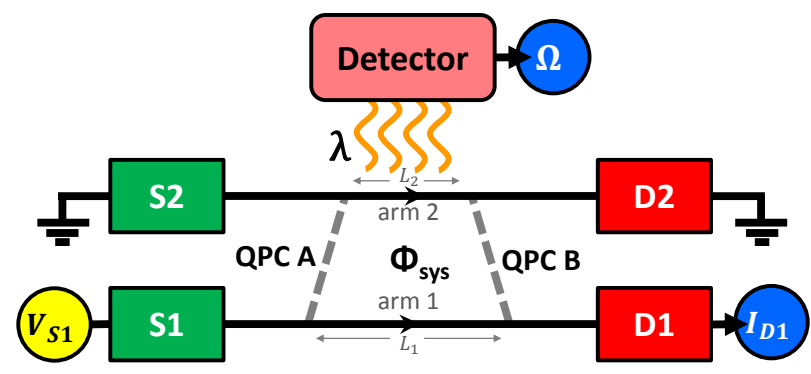

Fig. 1 (Color online) A weakly detected MZI. The average reading of the detector, $\Omega$, is linearly proportional (to leading order in system-detector coupling, $\lambda$ ) to the total electric charge on arm 2 of the interferometer at the time of the measurement. (If the detector is a current-carrying quantum point contact (QPC), then $\Omega(t)$ is equal to $\Omega(t) \sim \int_{t-\tau_{f l} / 2}^{t+\tau_{f l} / 2} I\left(t^{\prime}\right) d t^{\prime}$, where $I(t)$ is the current through the QPC and $\tau_{f l}=L_{2} / v$, with $L_{2}$ being the length of arm 2 and $v$ the velocity of the chiral edge mode). The strong follow-up measurement detects the charge (current pulse) arriving at the drain D1. Registering the outcome of the first measurement is subsequently conditioned on detecting a 'click' in $D 1$. This is the WV of the charge in arm 2.

outcome of the weak measurement of $A$ provided the result of the (strong) measurement of $B$ coincides with a prescribed value, $\langle A\rangle_{W V}=\frac{\operatorname{Tr}\left\{A \cdot \Pi_{B}\right\}}{\operatorname{Tr}\left\{\Pi_{B}\right\}}$, with $\Pi_{B}$ being the projection operator on the postselected subspace. WVs have been observed in experiments [18, 17, 11, 9. Their unusual expectation values [1] may be utilized for various purposes, including weak signal amplification [5, 22, 3, 23, 7, 29, quantum state discrimination [14, 30, and non-collapsing observation of virtual states [19]. There have been recent proposals to implement WV protocols in the context of solid state systems [21, 20, 6]. Apart from the realization with superconducting qubits in resonant cavity [9], setups made up of two (electrostatic interaction coupled) MZIs, operating in the quantum Hall regime, are of particular importance, given their immediate experimental availability [12, 25] and their versatile and controllable nature. In such setups one MZI plays the role of "the system", the other being "the detector".

Shpitalnik et al. 21 have implemented, in principle, a WV protocol in this setup, and have shown that the outcome of such measurement may produce a complete tomography of the WV measured in the system's MZI. An exhaustive analysis of the correlated signal in this system has been reported in the single particle regime 6], and the many body effects on the weak to strong measurement crossover have been classified 8. The fact that such a protocol is amenable to experimental verification 25] has been undermined by the lack of a concrete manual on how to implement it.

The present analysis is meant to point out that WVs in solid state physics setups are feasible. We point out, vis-à-vis a two MZI setup, which measurements need to be performed, how the sensitivity of the protocol can be controlled, how the readings of the detector should be calibrated, and consequently- how extreme WVs can be obtained and identified. 


\section{The system: a MZI}

Our system consists of a MZI depicted schematically in Fig. 1 . Electrons are injected from the source contact $S 1$, kept at a finite voltage bias $V_{S 1}$, and are collected at drain $D 1$. The other terminals of the interferometers are grounded. The Quantum Point Contacts (QPCs) A and B allow tunnelling of the electrons between arms 1 and 2 of the system; the electrons collected at $D 1$ are the result of the interference of different electron trajectories, and are sensitive to the magnetic flux $\Phi_{\text {sys }}$. A detector is electrostatically coupled to the charge in arm 2 of the interferometer. At this stage we refer to a general detector, weakly coupled to the system.

We consider explicitly the case where the bias current fed into the MZI is diluted (for example, one modifies the setup depicted in Fig. 1 such that most electrons emitted from the source $S 1$ are backscattered before arriving in the MZI). In that case, the width of an electron's wave packet is much smaller than the distance between two consecutive electrons. Moreover, we require that the time separation between successive injections of non-equilibrium electrons $\left(\tau_{V_{S 1}} \triangleq 2 \pi \hbar / e V_{S 1}\right.$ is much larger than the electron's time-of-flight through the interferometer's arm [27]: $\tau_{V_{S 1}} \gg \tau_{f l}$. To reduce adverse decoherence effects one may consider the limit of low temperature, low voltage bias, and nearly symmetric interferometers (i.e., nearly equal arm lengths). The conditions are met in actual experiments [12, 25.

The first step of our protocol consists of weakly measuring the electric charge $Q_{2}$, flowing through arm 2 of the interferometer. This (weak) measurement is performed as a snapshot at time $t_{W}$ of the electric charge along arm 2 . The followup (strong) measurement detects the charge arriving at the drain $D 1$ with a delay, $t_{\text {delay }}$, due to the finite propagation time of the charge from the weak detector to $D 1$. The measurement itself consists of integrating the current pulse over a window of time, $\left[t_{W}+t_{\text {delay }}, t_{W}+t_{\text {delay }}+\tau_{f l}\right]$, which corresponds to the time of flight of an electron through arm 2 (the latter is of length $L_{2} ; \tau_{f l}=L_{2} / v$, where $v$ is the Fermi velocity of the non-equilibrium electrons). We denote this integrated current by $\tau_{f l} I_{D 1}$. Under the conditions of diluted injected current specified above, the postselection signal, $I_{D 1}$, can reveal the detection of one or no electrons collected at $D 1$, we then condition the acceptance of the first measurement of $Q_{2}$ on a 'click' in $D 1$. Therefore the weak value of the charge on arm 2 is

$$
\left\langle Q_{2}\right\rangle_{W V}=\frac{\left\langle Q_{2}\left(t_{W}\right) \cdot I_{D 1}\left(t_{W}+t_{\text {delay }}+\frac{1}{2} \tau_{f l}\right)\right\rangle}{\left\langle I_{D 1}\right\rangle} .
$$

We now relate the abstract WV defined above to a measurable quantity. We assume that (to leading order in system-detector coupling) the average signal of the detector is linearly proportional to measured charge, i.e., $\langle\delta \Omega\rangle=\mathcal{S}\left\langle Q_{2}\right\rangle$, where $\Omega$ is the signal of the detector with $\delta \Omega \triangleq \Omega-\left.\Omega\right|_{\left\langle Q_{2}\right\rangle=0}$ and $\mathcal{S}$ is the sensitivity of the detector defined as

$$
\mathcal{S} \triangleq \frac{\partial\langle\Omega\rangle}{\partial\left\langle Q_{2}\right\rangle}
$$

We define the measured $\left\langle Q_{2}\right\rangle_{W V}^{M}$ as

$$
\left\langle Q_{2}\right\rangle_{W V}^{M} \triangleq \frac{1}{\mathcal{S}} \frac{\left\langle\delta \Omega \cdot I_{D 1}\right\rangle}{\left\langle I_{D 1}\right\rangle} .
$$




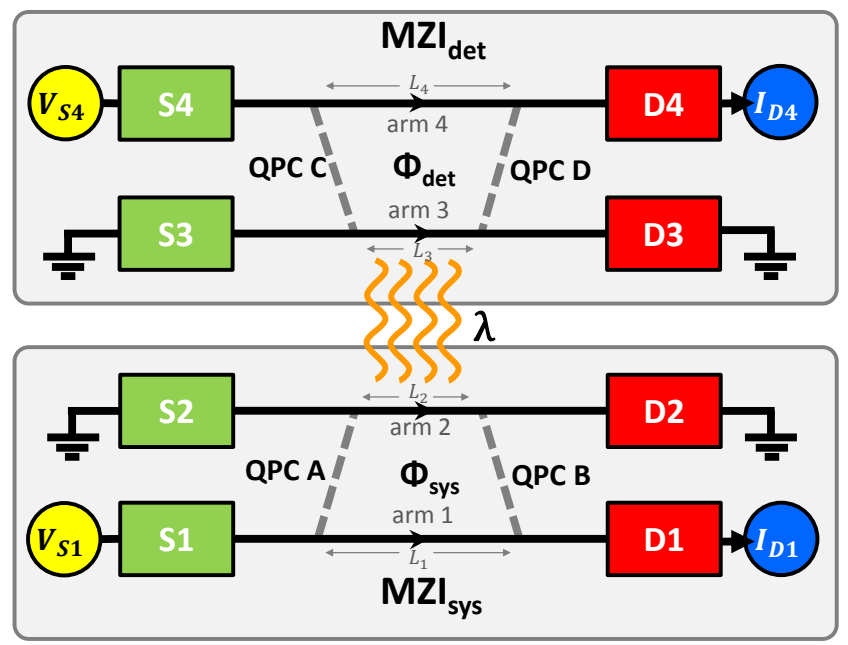

(a)

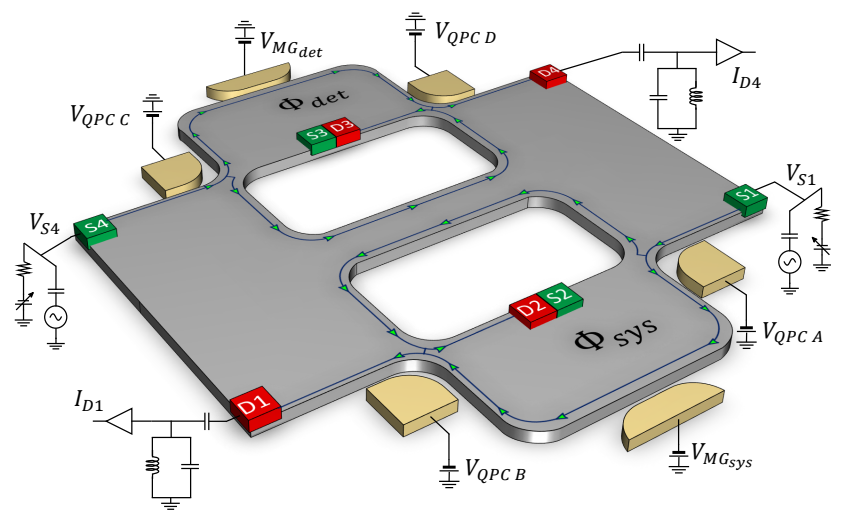

(b)

Fig. 2 (Color online) A concrete realization of the setup. (a) A schematic layout. (b) A realistic design. The system and the detector are realized by $\mathrm{MZI}_{\text {sys }}$ and $\mathrm{MZI}_{\mathrm{det}}$, which are weakly (with strength $\lambda$ ) electrostatically coupled through arms 2 and 3. The sources $S 1$ and $S 4$ are biased with the voltages $V_{S 1}$ and $V_{S 4}$ respectively, while the signals are measured in $D 1$ and $D 4$. The contacts $S 2, D 2, S 3$ and $D 3$ are connected to the ground. The gate voltages $V_{\text {QPC A-D }}$ control the inter-arm tunneling amplitudes of electrons near the respective QPC. The modulation gate biases $V_{M G_{s y s}}\left(V_{M G_{d e t}}\right)$ control the effective magnetic fluxes $\Phi_{s y s}\left(\Phi_{\text {det }}\right)$ by modifying the areas encircled by electronic trajectories along the device.

We expect the latter to be proportional to the WV $\left\langle Q_{2}\right\rangle_{W V}$ (c.f Eq. (1)).

\section{The detector: a MZI}

We realize the detector by $\mathrm{MZI}_{\mathrm{det}}$ as is shown in Fig. 2. Arm 3 of the detector is electrostatically weakly coupled to system's arm 2 . We assume that the detector 


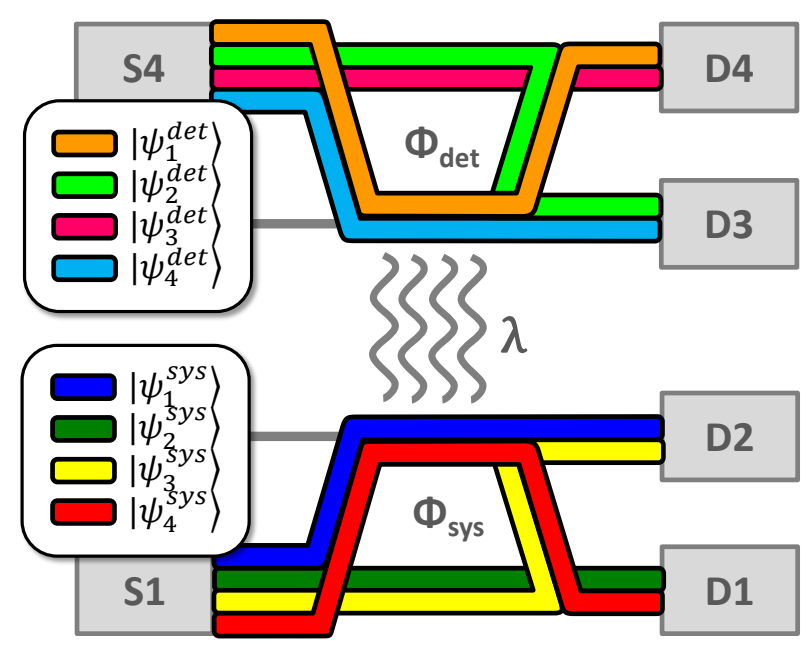

Fig. 3 (Color online) Electronic trajectories along MZIs. The underlying scattering electronic wavefunction can be constructed as an entangled product of electronic trajectories in $\mathrm{MZI}_{\text {sys }}$ and in $\mathrm{MZI}_{\mathrm{det}}$. Those are depicted schematically by different colors. cf. Appendix $\mathrm{A}$ for explicit expressions for the electronic trajectories.

MZI is tuned, analogously with the system MZI, to have a diluted incident current and a time-separation between incident electrons larger than the time-of-flight across the interferometer. The current of the detector integrated over $\tau_{f l}, \tau_{f l} I_{D 4}$, is sensitive to a charge on arm 2 , and serves as a pointer variable - It plays the role of $\Omega$ in the general formulation of the previos section. The (weak) signature of the system-detector interaction is a small additional phase gain of the wavefunction when a pair of electrons flow simultaneously along the arms 2 and 3 respectively 6 , 15 .

Therefore, the definition of $\left\langle Q_{2}\right\rangle_{W V}^{M}$ (Eq. (3)) in the case of MZI as a detector (cf. Fig. 2) reads

$$
\left\langle Q_{2}\right\rangle_{W V}^{M}=\frac{1}{\mathcal{S}}\left[\frac{\left\langle I_{D 4} I_{D 1}\right\rangle}{\left\langle I_{D 1}\right\rangle}-\left.\left\langle I_{D 4}\right\rangle\right|_{00}\right]
$$

It requires the measurement of the current-current correlator $\left\langle I_{D 4} I_{D 1}\right\rangle$, the average current in $D 1,\left\langle I_{D 1}\right\rangle$, and the average current in $D 4$ when the transmission of QPC A is set to zero, $\left.\left\langle I_{D 4}\right\rangle\right|_{00}$. The value of $\mathcal{S}$ is not known. It is evidently an essential element to determine whether the outcome of a weak value protocol in an experiment yields an anomalous (large) value. In order to determine $\mathcal{S}$, or equivalently to know the boundaries of the detector signal for unconditioned measurements, one needs a suitable calibration and characterization of the detector's sensitivity. 


\section{The system-detector coupling}

To gain physical insight on the detector's response and determine its proper calibration we resort to a two-particle picture (one electron passing through $\mathrm{MZI}_{\text {sys }}$ and another in $\left.\mathrm{MZI}_{\mathrm{det}}\right)$. The model is a valid description of the interaction between the system and detector electrons in the regime of diluted electron currents we are considering.

The two-particle scattering state, $\left|\Psi_{\text {sys,det }}\right\rangle$, near the drains (after the QPCs $\mathrm{B}$ and D) can be expressed in terms of partial electronic trajectories (cf. Fig. 3 ) as,

$$
\left|\Psi_{s y s, \text { det }}\right\rangle=\sum_{i j} e^{i \lambda_{i j}} c_{i}^{s y s}\left|\psi_{i}^{\text {sys }}\right\rangle c_{j}^{\text {det }}\left|\psi_{j}^{\text {det }}\right\rangle
$$

where $c_{i}^{\text {sys }}\left(c_{i}^{\text {det }}\right)$ are amplitudes of the trajectories through $\mathrm{MZI}_{\mathrm{sys}}\left(\mathrm{MZI}_{\mathrm{det}}\right)$ omitting the coupling between the interferometers (cf. Appendix A , and $\lambda_{i j}=\left\{\begin{array}{l}\lambda,(i=1 \vee 4) \wedge(j=1 \vee 4) \\ 0,\end{array}\right.$ is the weak coupling term $(\lambda \ll 1)$. The WV of $Q_{2}$ (cf. Eq. (1)) may be expressed in terms of the amplitudes $c_{i}$ as

$$
\left\langle Q_{2}\right\rangle_{W V}=\left\langle Q_{2}\right\rangle_{0} \frac{c_{4}^{\text {sys }}}{c_{4}^{\text {sys }}+c_{2}^{\text {sys }}} .
$$

where $\left\langle Q_{2}\right\rangle_{0} \approx \frac{e^{2} V_{S 1} L_{2}}{2 \pi \hbar v}$ is the average excess charge on the segment $L_{2}$. Similarly, we define

$$
\left\langle Q_{3}\right\rangle_{W V}=\left\langle Q_{3}\right\rangle_{0} \frac{c_{1}^{\text {det }}}{c_{1}^{\text {det }}+c_{3}^{\text {det }}},
$$

with $\left\langle Q_{3}\right\rangle_{0} \approx \frac{e^{2} V_{S 4} L_{3}}{2 \pi \hbar v}$, as the weak value of the charge on arm 3 conditioned on a signal in $D 4$. With these definitions the signal in $D 4$ to first order in $\lambda$ is given by (cf. Appendix B

$$
\left\langle I_{D 4}\right\rangle=\left\langle I_{D 4}\right\rangle_{0}\left(1+2 \tilde{\lambda} \mathfrak{I m}\left\{\left\langle Q_{3}\right\rangle_{W V}\left[\left\langle Q_{2}\right\rangle+\left\langle Q_{2}^{b g}\right\rangle\right]\right\}\right)
$$

where $\tilde{\lambda}=\lambda /\left\langle Q_{2}\right\rangle_{0}\left\langle Q_{3}\right\rangle_{0}$ and $\left\langle Q_{2}\right\rangle=\left\langle Q_{2}\right\rangle_{0}\left(\left|c_{1}^{s y s}\right|^{2}+\left|c_{4}^{\text {sys }}\right|^{2}\right)$ is the out-ofequilibrium and $\left\langle Q_{2}^{b g}\right\rangle$ is the background charge on arm $2,\left\langle I_{D 4}\right\rangle_{0}=\left(\left\langle Q_{3}\right\rangle_{0} / \tau_{f l}\right)\left|c_{1}^{\text {det }}+c_{3}^{\text {det }}\right|^{2}$ is the current measured at $D 4$ in the absence of interaction $(\lambda=0)$. We obtain also the explicit expression for Eq. (4) (cf. Apeendix B), given by

$$
\left\langle Q_{2}\right\rangle_{W V}^{M}=\frac{2 \tilde{\lambda}\left\langle I_{D 4}\right\rangle_{0}}{\mathcal{S}} \mathfrak{I m}\left\{\left\langle Q_{2}\right\rangle_{W V}\left(\left\langle Q_{3}\right\rangle_{W V}-\left\langle Q_{3}\right\rangle\right)\right\}
$$

From the analysis it also follows that the sensitivity of the MZI detector (cf. Eq. (2)) is

$$
\mathcal{S}=2 \tilde{\lambda}\left\langle I_{D 4}\right\rangle_{0} \mathfrak{I m}\left\{\left\langle Q_{3}\right\rangle_{W V}\right\}
$$

with the explicit expression $\mathcal{S}=2\left\langle Q_{3}\right\rangle_{0}^{2} \tilde{\lambda} / \tau_{f l}\left|c_{1}^{\text {det }} c_{3}^{\text {det }}\right| \sin \tilde{\phi}$. Here $\tilde{\phi}$ is the total phase difference between the trajectories $\left|\psi_{1}^{\text {det }}\right\rangle$ and $\left|\psi_{3}^{\text {det }}\right\rangle$ (cf. Fig. 3), including contributions from the $\mathrm{AB}$ flux, the orbital phase, and impurity scattering. As follows from eq. 10), the maximal sensitivity is obtained when (i) the QPCs C and $\mathrm{D}$ are set to half transmission and (ii) the total phase difference is $\tilde{\phi}=\pi / 2+\pi n$. 


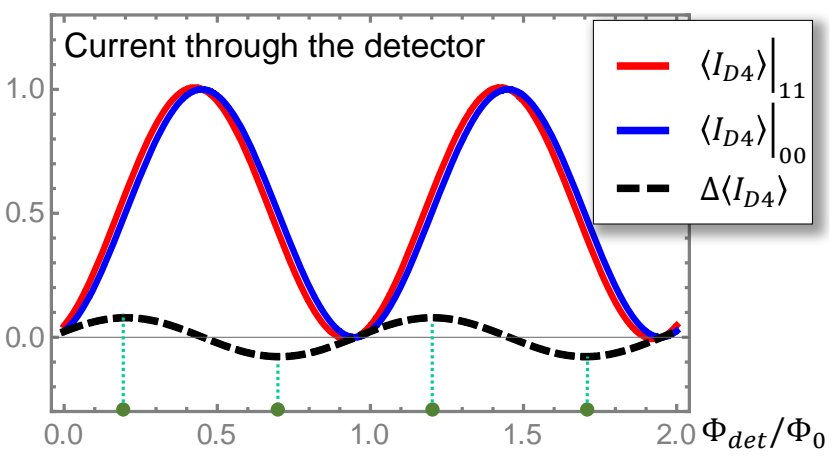

Fig. 4 (Color online) Calibration of the detector. The QPCs $\mathrm{C}$ and $\mathrm{D}$ are set to half transmission $\left(\left|t_{C}\right|=\left|t_{D}\right|=1 / \sqrt{2}\right), \tilde{\lambda}=0.05 \pi$. The currents are measured in units of $\left\langle Q_{3}\right\rangle_{0}$. The red (blue) curve corresponds to the signal in D4 when both QPC A and QPC B are fully transmitting (reflecting), $\left.\left\langle I_{D 4}\right\rangle\right|_{11}\left(\left.\left\langle I_{D 4}\right\rangle\right|_{00}\right)$. The black dashed curve is their difference: $\left.\Delta\left\langle I_{D 4}\right\rangle \triangleq\left\langle I_{D 4}\right\rangle\right|_{11}-\left.\left\langle I_{D 4}\right\rangle\right|_{00}$. The detector is maximally sensitive at the extremas of $\Delta\left\langle I_{D 4}\right\rangle$. Those points are highlighted by green dots.

The first requirement may be achieved by individually adjusting the gate voltages of the QPCs. To set the phase $\tilde{\phi}$ of the detector to the maximal sensitivity point we employ a calibration protocol discussed below.

\section{Calibration of the detector and extraction of weak values}

Let us assume the QPCs are already tuned to the half transmission point. Here we present a calibration protocol for the phase $\tilde{\phi}$ governing the interference signal registered in the detector. In the first step of this protocol, one sets QPC A of the system(!) (by tuning the gate voltage $V_{\mathrm{QPC}} \mathrm{A}$ ) to 'full reflection' (no current through both QPC A and QPC B 1 cf. Fig. 22, and measures the current $\left.\left\langle I_{D 4}\right\rangle\right|_{00}$ as function of the detector's flux $\Phi_{\text {det }} / \Phi_{0}$, where $\Phi_{0}=h / e$ is the magnetic flux quantum. Evidently the signal is independent of the system's flux $\Phi_{\text {sys }}$, and of the transmission of QPC B. In this configuration the amplitudes for trajectories $\left|\psi_{1}^{\text {sys }}\right\rangle$ and $\left|\psi_{4}^{\text {sys }}\right\rangle$ vanish and consequently $\left\langle Q_{2}\right\rangle=0$. In the second step both QPC A and QPC B are tuned to the opposite limit of 'full transmission' (a charge arriving from $S 1$ is deflected, with probability 1, to arm 2 (cf. Fig. 2)), setting the weight of the trajectories $\left|\psi_{2}^{\text {sys }}\right\rangle$ and $\left|\psi_{3}^{\text {sys }}\right\rangle$ to zero. In this configuration the current in $\mathrm{MZI}_{\text {sys }}$ flows through arm 2 and the charge $\left\langle Q_{2}\right\rangle$ reaches its maximal value, $\left\langle Q_{2}\right\rangle_{\max }$. In this limit, the current measured in the detector is denoted $\left.\left\langle I_{D 4}\right\rangle\right|_{11}$.

A representative plot of $\left\langle I_{D 4}\right\rangle$ for the two tunings: $\left.\left\langle I_{D 4}\right\rangle\right|_{00}$ and $\left.\left\langle I_{D 4}\right\rangle\right|_{1}$ respectively, and their difference $\left.\Delta\left\langle I_{D 4}\right\rangle \triangleq\left\langle I_{D 4}\right\rangle\right|_{11}-\left.\left\langle I_{D 4}\right\rangle\right|_{00}$ is shown in Fig. 4

\footnotetext{
${ }^{1}$ In fact, one can perform this calibration employing a general value of QPC B transmissivity.
} 
as a function of magnetic flux $\Phi_{\text {det }}$. The maximal sensitivity of the detector is achieved when one sets the magnetic flux to extremal points of $\Delta\left\langle I_{D 4}\right\rangle$. Values of the latter are depicted in the figure.

The calibration process prescribes the values of the sensitivity $\mathcal{S}$ (cf. Eq. (10)), $\left\langle Q_{3}\right\rangle_{W V}$ (cf. Eq. (7), $\left\langle I_{D 4}\right\rangle_{0}$ and $\left\langle Q_{3}\right\rangle=\left\langle Q_{3}\right\rangle_{0}\left(\left|c_{1}^{\text {det }}\right|^{2}+\left|c_{4}^{\text {det }}\right|^{2}\right.$ ) (cf. equations following Eq. (8p). It turns out that the calibration at maximal sensitivity yields $\mathfrak{R e}\left\{\left\langle Q_{3}\right\rangle_{W V}\right\}=\left\langle Q_{3}\right\rangle$. Using the latter equality and Eq. 10 we rewrite Eq. (9) as

$$
\left\langle Q_{2}\right\rangle_{W V}^{M}=\mathfrak{R e}\left\{\left\langle Q_{2}\right\rangle_{W V}\right\} \text { (at maximal sensitivity), }
$$

which sets the relation between the measured (real) quantity and the abstract definition of (complex) weak values. Consulting Eq. (4) we conclude that we can conveniently get rid of the factor $1 / \mathcal{S}$ by defining the normalized $\mathrm{WV}$,

$$
{\overline{\left\langle Q_{2}\right\rangle_{W V}^{M}}}_{W} \triangleq \frac{\left\langle Q_{2}\right\rangle_{W V}^{M}}{\left.\left\langle Q_{2}\right\rangle_{W V}^{M}\right|_{11}} .
$$

Here $\left.\left\langle Q_{2}\right\rangle_{W V}^{M}\right|_{11}$ is the measured $\left\langle Q_{2}\right\rangle_{W V}$, when both QPCs A and B are set to full transmission. For this tuning, it follows that $\left.\left\langle Q_{2}\right\rangle_{W V}^{M}\right|_{11}=\left\langle Q_{2}\right\rangle_{W V}=\left\langle Q_{2}\right\rangle_{\max }$. Eq. (12) involves only measurable quantities and serves to operatively identify weak values beyond the range allowed by unconditioned measurements.

\section{Results and discussion}

The present analysis is aimed at implementing the general framework of WV protocol [1] to a representative electronic system. The latter is experimentally accessible [25], rendering the present protocol amenable to experimental verification. We have focused on one central aspect of WV, namely the possibility to obtain expectation values (a.k.a. weak values) that lie beyond the range of possible outcomes of strong measurement [16] (the latter implies the collapse of the system's wave function). Specifically, our setup consists of a "system" and a "detector" $\left(\mathrm{MZI}_{\text {sys }}\right.$ and $\mathrm{MZI}_{\mathrm{det}}$, cf. Fig. 2) which are electrostatically weakly coupled. The detector is tuned to measure the charge transmitted through one of the system interferometer's arms (a weak "which path" measurement [2, 13, 10, 4]).

Intuition based on strong measurement procedure would suggest that when one electron is injected into the system's MZI, the normalized charge that can be measured on one of the interferometer arms is anything between 0 and 1 . By contrast, WV protocol allows us to obtain values which are above this value ("charge larger than 1" or even negative). The results shown in Fig. 5 make it clear that as far as weak values are concerned, both conventional values (that conform to "allowed values" of strong measurement) and exceptional values which lie beyond the interval $[0,1]$ are possible.

Specifically consider Figures 5 a and 5 b. We show that ${\overline{\left\langle Q_{2}\right.}}_{W V}^{M}$ may grow beyond the range of values allowed by a strong measurement of $Q_{2}$, which involves the collapse of the system's wavefunction. This is highlighted by a different (gray) color of the 3D plot. Figures 5 and 5 d show ${\overline{\left\langle Q_{2}\right\rangle_{W V}}}_{W V}^{M}$ along specific cuts of the 3D 


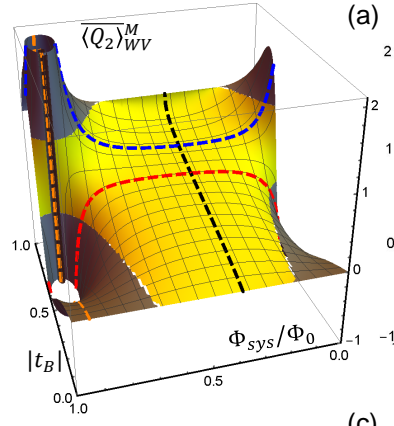

(a)

(b)
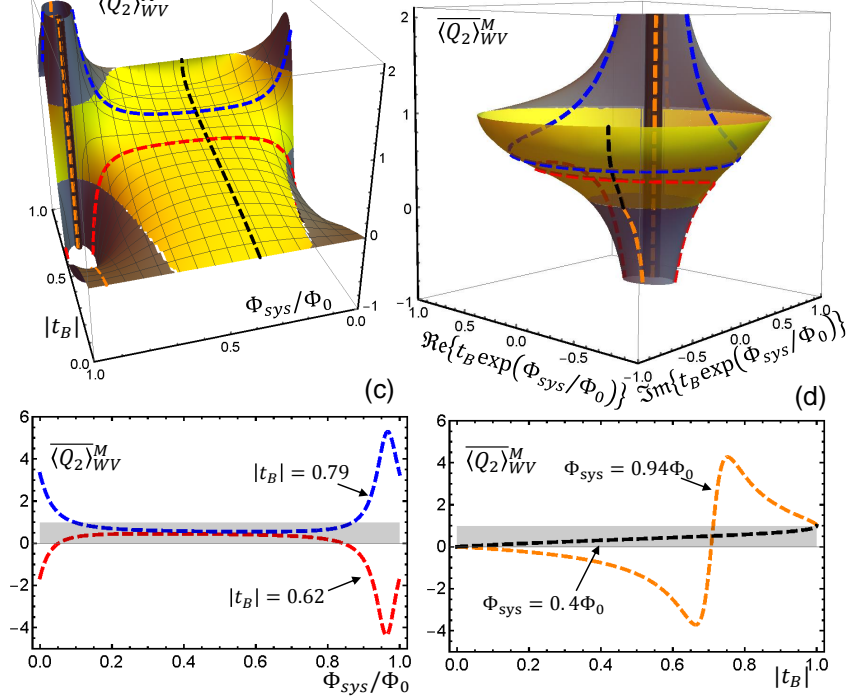

Fig. 5 (Color online) The normalized WV, ${\overline{\left\langle Q_{2}\right.}}_{W V}^{M}$, as a function of transmission amplitude of QPC B, $\left|t_{B}\right|$, and the flux, $\Phi_{s y s} / \Phi_{0}$. The transmission of QPC A is set to $\left|t_{A}\right|=\frac{1}{\sqrt{2}}$, and $\tilde{\lambda}=0.05 \pi$. An overall phase $\delta=0.1 \pi$, representing the asymmetry of the interferometer's arms is included, implying that the interference pattern is not symmetric around $\Phi_{0} / 2$. (a). A 3D plot of ${\overline{\left\langle Q_{2}\right\rangle_{W V}}}_{W}^{M}$ as a function of $t_{B}$ and $\Phi_{s y s} / \Phi_{0}$. (b). ${\overline{\left\langle Q_{2}\right\rangle_{W V}}}_{W}^{M}$ as a function of the real and the imaginary parts of $t_{B} \exp \left(\Phi_{s y s} / \Phi_{0}\right)$. The yellow colored region corresponds to values that fall within the conventional range, $[0,1]$, while the gray regions underline the observability of "exceptional values" that lie beyond this range. The dashed lines correspond to specific cuts along $\left|t_{B}\right|=0.62$ (red), $\left|t_{B}\right|=0.79$ (blue), $\Phi_{\text {sys }}=0.94 \Phi_{0}$ (orange), and $\Phi_{\text {sys }}=0.4 \Phi_{0}$ (black). Those are depicted in (c) vs. $\Phi_{s y s} / \Phi_{0}$ and in (d) vs. $\left|t_{B}\right|$, with the same respective colors.

plot, for $\left|t_{B}\right|=0.62$ and $\left|t_{B}\right|=0.79$ as a function of $\Phi_{\text {sys }} / \Phi_{0}$, and $\Phi_{\text {sys }}=0.94 \cdot \Phi_{0}$ and $\Phi_{\text {sys }}=0.4 \cdot \Phi_{0}$ as a function of $\left|t_{B}\right|$. We note that the WV may become exceedingly large (positive or negative) for a proper choice of the parameters.

In the procedure outlined above, we have put special emphasis on how the measured values of current and current correlations should be calibrated to fit with the weak value formalism. It would be interesting to repeat this analysis having in mind variations of our setup (e.g., replacing the MZI detector by a QPC or a current carrying quantum dot).

Acknowledgements We acknowledge discussions with H. Choi, M. Heiblum, I. Sivan, and E. Weisz. This work has been supported by Deutsche Forschungsgemeinschaft (DFG) grant RO 2247/8-1, SH 81/3-1, and RO 4710/1-1, the Israel Science Foundation (ISF), the Minerva foundation, and the Russia-Israel IMOS project. 


\section{A Appendix 1: Explicit expressions for electronic trajectories}

A.1 Solution to the single particle Hamiltonian

Here we solve the single-particle problem for a single MZI (suppose $\mathrm{MZI}_{\text {sys }}$ from figure 2 ) and expand the solution in partial electronic trajectories (cf. Fig. 33). We begin with the single particle Schrödinger equation

$$
\left(i \hbar \frac{\partial}{\partial t}-\hat{H}\right) \Psi=0
$$

where $\Psi=\left(\begin{array}{l}\psi_{1}(x, t) \\ \psi_{2}(x, t)\end{array}\right)$, and

$$
\left.\hat{H} \Psi=\left(\begin{array}{l}
i v \partial_{x} \psi_{1}(x, t)+\sum_{\alpha=A, B}\left[\frac{\Gamma_{\alpha}^{*}}{2} \delta\left(x-x_{1 \alpha}^{+}\right) \psi_{2}\left(x_{1 \alpha}^{-}, t\right)+\frac{\Gamma_{\alpha}^{*}}{2} \delta\left(x-x_{1 \alpha}^{-}\right) \psi_{2}\left(x_{1 \alpha}^{+}, t\right)\right. \\
i v \partial_{x} \psi_{2}(x, t)+\sum_{\alpha=A, B}\left[\frac{\Gamma_{\alpha}}{2} \delta\left(x-x_{2 \alpha}^{+}\right) \psi_{1}\left(x_{2 \alpha}^{-}, t\right)+\frac{\Gamma_{\alpha}}{2} \delta\left(x-x_{2 \alpha}^{-}\right) \psi_{1}\left(x_{2 \alpha}^{+}, t\right)\right.
\end{array}\right]\right) .
$$

Here $\psi_{1}(x, t)$ and $\psi_{2}(x, t)$ denote the wavefunctions in the corresponding arms 1 and $2, \Gamma_{\alpha}$ represents the tunneling term associated with the $\alpha$-th QPC, connecting points $x_{1 \alpha}$ and $x_{2 \alpha}$, $\alpha=A, B$ (cf. Fig. 6) $) ; x^{ \pm}=\lim _{\varepsilon \rightarrow 0} x \pm \varepsilon . \Gamma_{\alpha}$ may be related to the scattering amplitudes through Eqs. 16$)$

This problem is diagonal in the scattering basis

$$
\Psi_{k, l}(x, t)=\frac{1}{\sqrt{L}} e^{i k(x-v t)}\left\{\begin{array}{ll}
\nu_{l} & , x \in \mathrm{I} \\
\breve{S}_{A} \nu_{l} & , x \in \mathrm{II} \\
\breve{S}_{B} \nu_{l} & , x \in \mathrm{III}
\end{array} .\right.
$$

Here the Latin numerals denote the various sectors of the MZI: I - left to QPC A, II - between QPC A and B, III- right of QPC B, $\breve{S}_{\alpha}=\left(\begin{array}{cc}r_{\alpha} & -t_{\alpha}^{*} \\ t_{\alpha} & r_{\alpha}\end{array}\right)$ is the scattering matrix at QPC $\alpha$ and the scattering amplitudes are

$$
\begin{aligned}
r_{\alpha} & =\frac{\left(2 v^{2}\right)-\left|\Gamma_{\alpha}\right|^{2}}{\left(2 v^{2}\right)+\left|\Gamma_{\alpha}\right|^{2}} \\
t_{\alpha} & =\frac{4 i v \Gamma_{\alpha}}{\left(2 v^{2}\right)+\left|\Gamma_{\alpha}\right|^{2}},
\end{aligned}
$$

for the symmetric MZI case $\left(x_{1 B}-x_{1 A}=x_{2 B}-x_{2 A}\right)$. The index $l=1,2$ denotes two orthogonal solutions $\boldsymbol{\nu}_{1}=\left(\begin{array}{l}1 \\ 0\end{array}\right)$ and $\boldsymbol{\nu}_{2}=\left(\begin{array}{l}0 \\ 1\end{array}\right)$ that correspond to the scattering state incident from $S 1$ or $S 2$.

A.2 Explicit expressions for the coefficients $c_{i}$

The probability of the particle incident from $S 1$ to be detected in $D 1$ can be presented using the path integral formalism as

$$
P_{S 1 \rightarrow D 1}=\left|\int_{\mathcal{C}} \mathcal{D} \Psi e^{i S\{\Psi\} / \hbar}\right|^{2},
$$

where $\mathcal{C}$ represents all the trajectories from $S 1$ to $D 1$, and $c_{i} \triangleq e^{i S\left\{\Psi_{i}\right\} / \hbar}$ is the weight of the corresponding trajectory. The same argument may be repeated for probabilities $P_{S 1 \rightarrow D 2}$, $P_{S 2 \rightarrow D 1}$, and $P_{S 2 \rightarrow D 2}$ to include all the trajectories depicted in Fig. 3 The explicit expressions for the trajectory weights, $c_{i}$, may be found from the exact solution, Eq. 15. Here we 


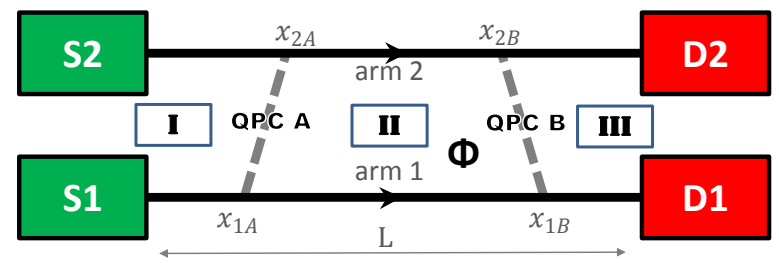

Fig. 6 (Color online) A schematics of a MZI. The points $x_{1 \alpha}$ and $x_{2 \alpha}, \alpha=A, B$ at QPCs $\mathrm{A}$ and $\mathrm{B}$ are connected by the tunneling terms $\Gamma_{\alpha}$. The Latin numerals denote the various sectors of the MZI: I - left to QPC A, II - between QPC A and B, III- right of QPC B.

summarize the results. Up to unimportant orbital phases

$$
\begin{aligned}
c_{1}^{\text {sys }} & =-t_{A}^{*} r_{B} \\
c_{2}^{\text {sys }} & =r_{A} r_{B} \\
c_{3}^{\text {sys }} & =-r_{A} t_{B}^{*} \\
c_{4}^{\text {sys }} & =-t_{A}^{*} t_{B} \\
c_{1}^{\text {det }} & =-t_{C} t_{D}^{*} \\
c_{2}^{\text {det }} & =r_{C} t_{D} \\
c_{3}^{\text {det }} & =r_{C} r_{D} \\
c_{4}^{\text {det }} & =t_{C} r_{D} .
\end{aligned}
$$

\section{B Appendix 2: Derivation of expectation values and correlators}

Here we derive explicit expressions for expectation values of operators that appear throughout the manuscript. For simplicity we assume a symmetric MZI $\left(L_{1}=L_{2}, L_{3}=L_{4}\right)$ operating in the low frequency, zero temperature regime, where the quantum state does not vary much over the time of the experiment, hence is almost steady. Thus, in this regime, all quantities are essentially time independent. We evaluate the expectation values by computing a trace of the operator with respect to the initial density matrix, $\rho_{i}=|S 1, S 4\rangle\langle S 1, S 4|$ describing two particles, which are taken fom out-of equilibrium distribution, and which are incident from the biased sources $S 1$ and $S 4$ (cf. Fig. 2). At the end of this appendix (cf. Section B.4) we add a contribution from the equilibrium, background sea of electrons below the Fermi level. Throughout this section, the charge operators associated with each MZI are normalized to have no physical units. The latter may be recovered at the end, multiplying the normalized charge by $\left\langle Q_{i}\right\rangle_{0}(i=2,3)$ (those are defined following Eqs. (6) and (7)).

B.1 Derivation of equations (6) and (7)

Writing explicitly the expectation values appearing in eq. 11), we come up with the expression

$$
\left\langle Q_{2}\right\rangle_{W V}=\frac{\left\langle S 1, S 4\left|Q_{2} I_{D 1}\right| S 1, S 4\right\rangle}{\left\langle S 1, S 4\left|I_{D 1}\right| S 1, S 4\right\rangle}
$$

where $I_{D 1}=|D 1\rangle\langle D 1|$. To leading order in the coupling, $\lambda$ (cf. eq. (5)), the two MZIs may be considered as decoupled, and the trace over $\mathrm{MZI}_{\text {det }}$ states $(|S 4\rangle\langle S 4|)$ is trivial. Hence the expression reads

$$
\left\langle Q_{2}\right\rangle_{W V}=\frac{\left\langle S 1\left|Q_{2}\right| D 1\right\rangle}{\langle S 1 \mid D 1\rangle}
$$


The operator $Q_{2}$ is proportional to a projection operator that selects only partial wavepackets $\left|\psi_{\text {sys }}^{1}\right\rangle$ and $\left|\psi_{\text {sys }}^{4}\right\rangle$ (cf. Fig. 3. Only $\left|\psi_{\text {sys }}^{1}\right\rangle$ has support at D1 and contributes to the numerator. The denominator includes two contributions, $\left|\psi_{\text {sys }}^{2}\right\rangle$ and $\left|\psi_{\text {sys }}^{4}\right\rangle$. It follows that

$$
\left\langle Q_{2}\right\rangle_{W V}=\frac{c_{4}^{s y s}}{c_{4}^{s y s}+c_{2}^{s y s}} .
$$

The derivation of an expression for $\left\langle Q_{3}\right\rangle_{W V} \triangleq \frac{\left\langle Q_{3} \cdot I_{D 4}\right\rangle}{\left\langle I_{D 4}\right\rangle}$ (eq. [7) is similar.

\section{B.2 Expression for the system-detector current correlator}

Here we derive the expression for $\left\langle I_{D 1} I_{D 4}\right\rangle$ to leading order in the coupling $\lambda$. The expression for the non-equilibrium currents correlator reads

$$
\left\langle I_{D 1} I_{D 4}\right\rangle=\left\langle S 1, S 4\left|I_{D 1} I_{D 4}\right| S 1, S 4\right\rangle .
$$

We plug in the explicit expressions for the currents, $I_{D 1}=|D 1\rangle\langle D 1|$ and $I_{D 4}=|D 4\rangle\langle D 4|$ to obtain,

$$
\left\langle I_{D 1} I_{D 4}\right\rangle=|\langle S 1, S 4 \mid D 1, D 4\rangle|^{2} .
$$

We note that due to charge conservation and the assumption of a steady state, the sum of the currents on the two arms (between the QPCs) of any MZI, is equal to the total current that flows into the MZI. For example, for $\mathrm{MZI}_{\text {sys }}$,

$$
I_{S 1}=I_{1}+I_{2}
$$

where $I_{S 1}$ denotes an operator that measures current flow through $S 1$, and $I_{1(2)}$ measure currents at arbitrary points on arm 1 (2) (cf. Fig 2). Next, we integrate Eq. 24) over the timeof flight of electron in the MZI. Integration of $I_{S 1}$ over this time yields the total charge that flows into the MZI during the time-of-flight. The latter is noiseless according to our assumptions and therefore is proportional to the identity (exactly one electron has been injected from $S 1$ ). The integration of $I_{1(2)}$ yields the fraction of charge that when to arm $1(2), Q_{1(2)}$. It follows from the above that

$$
Q_{1}+Q_{2}=\mathbb{1},
$$

where we have used $\tau_{f l} I_{S 1}=\mathbb{1}$ in dimensionless units. A similar identity may be obtained for $\mathrm{MZI}_{\mathrm{det}}$,

$$
Q_{3}+Q_{4}=\mathbb{1} \text {. }
$$

Next, we insert those unit operators into Eq. (23), which yields the expression

$$
\begin{aligned}
& \left\langle I_{D 1} I_{D 4}\right\rangle=\mid\langle S 1, S 4| Q_{1} Q_{3}+Q_{1} Q_{4}+ \\
& +Q_{2} Q_{3}+\left.Q_{2} Q_{4}|D 1, D 4\rangle\right|^{2} .
\end{aligned}
$$

Each element in the sum is diagonal in the basis of trajectories, and can be evaluated employing the wavefunction (5). It follows that

$$
\begin{aligned}
\left\langle I_{D 1} I_{D 4}\right\rangle= & \mid\left\langle Q_{1}\right\rangle_{S 1 ; D 1}\left\langle Q_{3}\right\rangle_{S 4 ; D 4}+\left\langle Q_{1}\right\rangle_{S 1 ; D 1}\left\langle Q_{4}\right\rangle_{S 4 ; D 4}+ \\
& +e^{i \lambda}\left\langle Q_{2}\right\rangle_{S 1 ; D 1}\left\langle Q_{3}\right\rangle_{S 4 ; D 4}+\left.\left\langle Q_{2}\right\rangle_{S 1 ; D 1}\left\langle Q_{4}\right\rangle_{S 4 ; D 4}\right|^{2},
\end{aligned}
$$

where we have introduced the notation $\langle Q\rangle_{S ; D}=\langle S|Q| D\rangle$. Using the identities 25, and 26, and expanding to leading order in $\lambda$, we arrive at the expression

$$
\left\langle I_{D 1} I_{D 4}\right\rangle=\left|\langle\mathbb{1}\rangle_{S 1 ; D 1}\langle\mathbb{1}\rangle_{S 4 ; D 4}+i \lambda\left\langle Q_{2}\right\rangle_{S 1 ; D 1}\left\langle Q_{3}\right\rangle_{S 4 ; D 4}\right|^{2} .
$$

Simplifying it, employing the relations $\left\langle I_{D 1}\right\rangle_{0} \equiv\left|\langle\mathbb{1}\rangle_{S 1 ; D 1}\right|^{2},\left\langle I_{D 4}\right\rangle_{0} \equiv\left|\langle\mathbb{1}\rangle_{S 4 ; D 4}\right|^{2}$ (see for example Eq. 23 and eq. 20), we obtain

$$
\left\langle I_{D 1} I_{D 4}\right\rangle=\left\langle I_{D 1}\right\rangle_{0}\left\langle I_{D 4}\right\rangle_{0}\left(1+2 \lambda \mathfrak{I m}\left\{\left\langle Q_{2}\right\rangle_{W V}\left\langle Q_{3}\right\rangle_{W V}\right\}\right) .
$$


B.3 Expressions for the current expectation values

Here we compute the expressions for the current expectation values. To do so, we employ the charge conservation in a steady state regime. We repeat the discussion following Eq. 25 that led to the identities

$$
\begin{aligned}
& I_{D 1}+I_{D 2}=\mathbb{1} \\
& I_{D 3}+I_{D 4}=\mathbb{1} .
\end{aligned}
$$

The latter may be employed to write

$$
\begin{aligned}
& \left\langle I_{D 1}\right\rangle=\left\langle I_{D 1} I_{D 3}\right\rangle+\left\langle I_{D 1} I_{D 4}\right\rangle \\
& \left\langle I_{D 4}\right\rangle=\left\langle I_{D 2} I_{D 4}\right\rangle+\left\langle I_{D 1} I_{D 4}\right\rangle .
\end{aligned}
$$

Since $\left\langle I_{D 1} I_{D 4}\right\rangle$ was found in eq. 30 , one may follow the derivation in appendix B.2 to obtain an expression for $\left\langle I_{D 1} I_{D 3}\right\rangle$ and $\left\langle I_{D 2} I_{D 4}\right\rangle$. Plugging those results into Eq. 32 one ends up with the equalities

$$
\begin{aligned}
& \left\langle I_{D 1}\right\rangle=\left\langle I_{D 1}\right\rangle_{0}\left(1+2 \lambda \mathfrak{I m}\left\{\left\langle Q_{2}\right\rangle_{W V}\left\langle Q_{3}\right\rangle\right\}\right) \\
& \left\langle I_{D 4}\right\rangle=\left\langle I_{D 4}\right\rangle_{0}\left(1+2 \lambda \mathfrak{I m}\left\{\left\langle Q_{3}\right\rangle_{W V}\left\langle Q_{2}\right\rangle\right\}\right) .
\end{aligned}
$$

B.4 The contribution of the background charge

Above we have derived expressions for the average currents and current-current correlator in the presence of single particle taken from out-of-equilibrium distribution. In real life, there is background charge. The latter may interact with the incoming electrons, producing a shift in the measured signal. We consider the background charge as a noiseless constant that shifts the charge operator on each arm $\left(Q_{2} \rightarrow Q_{2}+\left\langle Q_{2}^{b g}\right\rangle, Q_{3} \rightarrow Q_{3}+\left\langle Q_{3}^{b g}\right\rangle\right)$. We may now rewrite now eqs. 30 and 33 with the contribution of the background charge,

$$
\begin{gathered}
\left\langle I_{D 1}\right\rangle=\left\langle I_{D 1}\right\rangle_{0}\left(1+2 \lambda \mathfrak{I m}\left\{\left\langle Q_{2}\right\rangle_{W V}\left[\left\langle Q_{3}\right\rangle+\left\langle Q_{3}^{b g}\right\rangle\right]\right\}\right) \\
\left\langle I_{D 4}\right\rangle=\left\langle I_{D 4}\right\rangle_{0}\left(1+2 \lambda \mathfrak{I m}\left\{\left\langle Q_{3}\right\rangle_{W V}\left[\left\langle Q_{2}\right\rangle+\left\langle Q_{2}^{b g}\right\rangle\right]\right\}\right) \\
\left\langle I_{D 4} I_{D 1}\right\rangle=\left\langle I_{D 4}\right\rangle_{0}\left\langle I_{D 1}\right\rangle_{0}\left[1+2 \lambda \mathfrak{m}\left\{\left[\left\langle Q_{2}\right\rangle_{W V}+\left\langle Q_{2}^{b g}\right\rangle\right]\left[\left\langle Q_{3}\right\rangle_{W V}+\left\langle Q_{3}^{b g}\right\rangle\right]\right\}\right]
\end{gathered}
$$

(cf. Eq. (8)).

\section{References}

1. Y. Aharonov, D.Z. Albert, L. Vaidman, How the result of a measurement of a component of the spin of a spin- 1/2 particle can turn out to be 100. Phys. Rev. Lett. 60(14) (1988). doi:10.1103/PhysRevLett.60.1351

2. I.L. Aleiner, N.S. Wingreen, Y. Meir, Dephasing and the Orthogonality Catastrophe in Tunneling through a Quantum Dot: The Which Path? Interferometer. Phys. Rev. Lett. 79(19) (1997). doi:10.1103/PhysRevLett.79.3740

3. N. Brunner, C. Simon, Measuring Small Longitudinal Phase Shifts: Weak Measurements or Standard Interferometry? Phys. Rev. Lett. 105(1), 010405 (2010). doi:10.1103/PhysRevLett.105.010405

4. E. Buks, R. Schuster, M. Heiblum, D. Mahalu, V. Umansky, Dephasing in electron interference by a "which-path" detector. Nature 391(6670), 871-874 (1998). doi:10.1038/36057 
5. P.B. Dixon, D.J. Starling, A.N. Jordan, J.C. Howell, Ultrasensitive Beam Deflection Measurement via Interferometric Weak Value Amplification. Phys. Rev. Lett. 102(17), 173601 (2009). doi:10.1103/PhysRevLett.102.173601

6. J. Dressel, Y. Choi, A.N. Jordan, Measuring which-path information with coupled electronic Mach-Zehnder interferometers. Phys. Rev. B 85(4), 045320 (2012). doi:10.1103/PhysRevB.85.045320

7. J. Dressel, M. Malik, F.M. Miatto, A.N. Jordan, R.W. Boyd, Colloquium : Understanding quantum weak values: Basics and applications. Rev. Mod. Phys. 86(1), 307-316 (2014). doi:10.1103/RevModPhys.86.307

8. I. Esin, A. Romito, Y.M. Blanter, Y. Gefen, Crossover between strong and weak measurement in interacting many-body systems. New. J. Phys. 18(1), 013016 (2016). doi:10.1088/1367-2630/18/1/013016

9. J.P. Groen, D. Ristè, L. Tornberg, J. Cramer, P.C. de Groot, T. Picot, G. Johansson, L. DiCarlo, Partial-Measurement Backaction and Nonclassical Weak Values in a Superconducting Circuit. Phys. Rev. Lett. $111(9), 090506$ (2013). doi:10.1103/PhysRevLett.111.090506

10. S.A. Gurvitz, Measurements with a noninvasive detector and dephasing mechanism. Phys. Rev. B 56(23), 15215-15223 (1997). doi:10.1103/PhysRevB.56.15215

11. O. Hosten, P. Kwiat, Observation of the spin hall effect of light via weak measurements. Science 319, 787-90 (2008). doi:10.1126/science.1152697

12. Y. Ji, Y. Chung, D. Sprinzak, M. Heiblum, D. Mahalu, H. Shtrikman, An electronic MachZehnder interferometer. Nature 422, 415-418 (2003). doi:10.1038/nature01503

13. Y. Levinson, Dephasing in a quantum dot due to coupling with a quantum point contact. Eur. Phys. Lett. 39(3), 299-304 (1997). doi:10.1209/epl/i1997-00351-x

14. J.S. Lundeen, B. Sutherland, A. Patel, C. Stewart, C. Bamber, Direct measurement of the quantum wavefunction. Nature 474, 188-91 (2011). doi:10.1038/nature10120

15. I. Neder, M. Heiblum, D. Mahalu, V. Umansky, Entanglement, Dephasing, and Phase Recovery via Cross-Correlation Measurements of Electrons. Phys. Rev. Lett. 98(3), 036803 (2007). doi:10.1103/PhysRevLett.98.036803

16. J.V. Neumann, Mathematical Foundations of Quantum Mechanics (Investigations in physics (Princeton University Press), Princeton). 0691028931

17. G.J. Pryde, J.L. OBrien, A.G. White, T.C. Ralph, H.M. Wiseman, Measurement of Quantum Weak Values of Photon Polarization. Phys. Rev. Lett. 94(22), 220405 (2005). doi:10.1103/PhysRevLett.94.220405

18. N.W.M. Ritchie, J.G. Story, R.G. Hulet, Realization of a measurement of a "weak value". Phys. Rev. Lett. 66(9), 1107-1110 (1991). doi:10.1103/PhysRevLett.66.1107

19. A. Romito, Y. Gefen, Weak measurement of cotunneling time. Phys. Rev. B 90(8), 085417 (2014). doi:10.1103/PhysRevB.90.085417

20. A. Romito, Y. Gefen, Y.M. Blanter, Weak Values of Electron Spin in a Double Quantum Dot. Phys. Rev. Lett. 100(5), 056801 (2008). doi:10.1103/PhysRevLett.100.056801

21. V. Shpitalnik, Y. Gefen, A. Romito, Tomography of Many-Body Weak Values: Mach-Zehnder Interferometry. Phys. Rev. Lett. 101(22), 226802 (2008). doi:10.1103/PhysRevLett.101.226802

22. D.J. Starling, P.B. Dixon, A.N. Jordan, J.C. Howell, Optimizing the signal-to-noise ratio of a beam-deflection measurement with interferometric weak values. Phys. Rev. A 80(4), 041803 (2009). doi:10.1103/PhysRevA.80.041803

23. D.J. Starling, P.B. Dixon, N.S. Williams, A.N. Jordan, J.C. Howell, Continuous phase amplification with a Sagnac interferometer. Phys. Rev. A 82(1), 011802 (2010). doi:10.1103/PhysRevA.82.011802

24. R. Vijay, C. Macklin, D.H. Slichter, S.J. Weber, K.W. Murch, R. Naik, A.N. Korotkov, I. Siddiqi, Stabilizing Rabi oscillations in a superconducting qubit using quantum feedback. Nature 490, 77-80 (2012). doi:10.1038/nature11505

25. E. Weisz, H.K. Choi, I. Sivan, M. Heiblum, Y. Gefen, D. Mahalu, V. Umansky, An electronic quantum eraser. Science 344(6190), 1363-6 (2014). doi:10.1126/science.1248459

26. H.M. Wiseman, G.J. Milburn, Quantum Measurement and Control (Cambridge University Press, Cambridge, 2010). ISBN 0521804426

27. S.-C. Youn, H.-W. Lee, H.-S. Sim, Nonequilibrium Dephasing in an Electronic Mach-Zehnder Interferometer. Phys. Rev. Lett. 100(19), 196807 (2008). doi:10.1103/PhysRevLett.100.196807

28. Q. Zhang, R. Ruskov, A.N. Korotkov, Continuous quantum feedback of coherent oscillations in a solid-state qubit. Phys. Rev. B 72(24), 245322 (2005). 
doi:10.1103/PhysRevB.72.245322

29. O. Zilberberg, A. Romito, Y. Gefen, Charge Sensing Amplification via Weak Values Measurement. Phys. Rev. Lett. 106(8), 080405 (2011). doi:10.1103/PhysRevLett.106.080405

30. O. Zilberberg, A. Romito, D.J. Starling, G.A. Howland, C.J. Broadbent, J.C. Howell, Y. Gefen, Null Values and Quantum State Discrìmination. Phys. Rev. Lett. 110(17), 170405 (2013). doi:10.1103/PhysRevLett.110.170405 